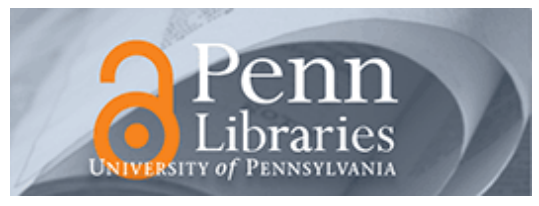

University of Pennsylvania ScholarlyCommons

October 2007

\title{
Higher-Order Resonant Power Flow Inside and Around Superdirective Plasmonic Nanoparticles
}

Andrea Alù

University of Pennsylvania, andreaal@seas.upenn.edu

Nader Engheta

University of Pennsylvania, engheta@seas.upenn.edu

Follow this and additional works at: https://repository.upenn.edu/ese_papers

\section{Recommended Citation}

Andrea Alù and Nader Engheta, "Higher-Order Resonant Power Flow Inside and Around Superdirective Plasmonic Nanoparticles", . October 2007.

Copyright 2007 Optical Society of America. Postprint version. Published in Journal of the Optical Society of America B - Optical Physics, Volume 24, Issue 10, October 2007, pages A89-A97.

Publisher URL: http://dx.doi.org/10.1364/JOSAB.24.000A89

This paper is posted at ScholarlyCommons. https://repository.upenn.edu/ese_papers/437

For more information, please contact repository@pobox.upenn.edu. 


\title{
Higher-Order Resonant Power Flow Inside and Around Superdirective Plasmonic Nanoparticles
}

\author{
Abstract \\ The optical power flow around a plasmonic particle has been a topic of research interest over the years \\ [see e.g., C. F. Bohren, Am. J. Phys. 51, 323 (1983); V. A. Bashevoy, V. A. Federov, and N. I. Zheludev, Optics \\ Express, 13, 8372 (2005)]. Here we revisit this problem with an emphasis on higher-order resonances, and \\ we present the theoretical results of our analysis for such power flow distribution for plasmonic \\ nanoparticles at their multipolar resonances. Results for the second and third orders of resonances show \\ optical power flow patterns that are significantly different from that of the first-order resonance inside and \\ around plasmonic superdirective nanoparticles, with multi-center vortices, saddle points and saddle lines, \\ and with an anomalous circulation of power resembling higher-order modes in a resonant cavity. A \\ potential application of these optical flow patterns to trap or move a neighboring nanoparticle is also \\ briefly suggested.
}

\section{Keywords}

metals, surface plasmons, mie theory

\section{Comments}

Copyright 2007 Optical Society of America. Postprint version. Published in Journal of the Optical Society of America B - Optical Physics, Volume 24, Issue 10, October 2007, pages A89-A97.

Publisher URL: http://dx.doi.org/10.1364/JOSAB.24.000A89 


\title{
Higher-Order Resonant Power Flow Inside and Around Superdirective Plasmonic Nanoparticles
}

\author{
Andrea Alù and Nader Engheta \\ University of Pennsylvania, Dept. of Electrical and Systems Engineering, Phildelphia, \\ PA, 19104, U.S.A.
}

The optical power flow around a plasmonic particle has been a topic of research interest over the years [see e.g., C. F. Bohren, Am. J. Phys. 51, 323 (1983); V. A. Bashevoy, V. A. Federov, and N. I. Zheludev, Optics Express, 13, 8372 (2005)]. Here we revisit this problem with an emphasis on higher-order resonances, and we present the theoretical results of our analysis for such power flow distribution for plasmonic nanoparticles at their multipolar resonances. Results for the second and third orders of resonances show optical power flow patterns that are significantly different from that of the first-order resonance inside and around plasmonic superdirective nanoparticles, with multi-center vortices, saddle points and saddle lines, and with an anomalous circulation of power resembling higher-order modes in a resonant cavity. A potential application of these optical flow patterns to trap or move a neighboring nanoparticle is also briefly suggested.@ 2007 Optical Society of America

OCIS Codes: 250.5420, 160.3900, 240.6680, 290.4020 


\section{Introduction}

The characteristics of light interaction with plasmonic nanoparticles have been studied for many years, and it has been known that the local plasmonic resonances are responsible for certain optical phenomena [1] such as the shining colors of noble metals, Raman scattering and Wood anomalies, to name a few (see [2] and references therein). Although the physical phenomena underlying these effects are widely understood and complete theories and analyses are available for the canonical geometries, studies and interests in nano-resonant systems and plasmonic materials have increased in recent years from the theoretical and experimental points of view. This is mainly due to the recent interest in use of nano-scale metamaterials and plasmonic structures, e.g., sub-wavelength imaging [3], sub-diffractive guided-wave propagation [4], optical nanocircuit elements [5] and electromagnetic transparency and cloaking [6-8]. As another possible use of plasmonic resonances, the idea of exciting higher-order resonances in a sub-wavelength system for super-directive nano-radiators at infrared and optical frequencies has been recently proposed by our group [9].

The fundamental theory explaining the physics behind the resonances of plasmonic subwavelength particles is based on Mie's seminal work [10]. The question of optical power flow around the sub-wavelength plasmonic particles at their dipolar resonance has been considered for some time by various groups [11-13]. Here, we revisit this issue using the full-wave analysis in the Mie theory, but with particular attention to the peculiarities of such power flow for the higher-order resonant particles. 
As is well known from the Mie theory, the scattering from a spherical particle embedded in free space and excited by an $e^{-i \omega t}$ field distribution may be evaluated in exact form by expanding the impinging and scattered fields in terms of spherical harmonics. For the impinging field we have:

$$
\begin{aligned}
\mathbf{E}_{i} & =E_{0}\left(\sum_{n, m} a_{n m}^{T M} \mathbf{N}_{n m}^{(1)}+\sum_{n, m} a_{n m}^{T E} \mathbf{M}_{n m}^{(1)}\right) \\
\mathbf{H}_{i} & =-i \frac{E_{0}}{\eta_{0}}\left(\sum_{n, m} a_{n m}^{T E} \mathbf{N}_{n m}^{(1)}+\sum_{n, m} a_{n m}^{T M} \mathbf{M}_{n m}^{(1)}\right)^{\prime}
\end{aligned}
$$

where $\mathbf{M}_{n m}^{(1)}$ and $\mathbf{N}_{n m}^{(1)}$ are orthogonal vector spherical harmonics, as defined in [14], whose analytical expression contains spherical Bessel $j_{n}$ functions for the radial variation of the field, $E_{0}$ is the arbitrary electric field amplitude and $\eta_{0}$ is the free-space characteristic impedance. The coefficients $a_{n m}$ are determined from the impinging field and are evaluated in closed form for plane wave incidence or other canonical forms of excitation [14]. The scattered field may be expanded analogous to (1), but employing $\mathbf{M}_{n m}^{(3)}$ and $\mathbf{N}_{n m}^{(3)}$ vector spherical harmonics, containing spherical Hankel functions $h_{n}^{(1)}$ in order to satisfy the radiation condition at infinity:

$$
\begin{aligned}
& \mathbf{E}_{s}=E_{0}\left(\sum_{n, m} c_{n}^{T M} a_{n m}^{T M} \mathbf{N}_{n m}^{(3)}+\sum_{n, m} c_{n}^{T E} a_{n m}^{T E} \mathbf{M}_{n m}^{(3)}\right) \\
& \mathbf{H}_{s}=-i \frac{E_{0}}{\eta_{0}}\left(\sum_{n, m} c_{n}^{T E} a_{n m}^{T E} \mathbf{N}_{n m}^{(3)}+\sum_{n, m} c_{n}^{T M} a_{n m}^{T M} \mathbf{M}_{n m}^{(3)}\right)
\end{aligned}
$$

As is well known, owing to the orthogonality of the spherical harmonics, each $T E^{r}{ }_{n m}$ or $T M^{r}{ }_{n m}$ scattered wave in (2) has an amplitude proportional to the one of the 
corresponding impinging harmonic through the unknown scattering coefficient $c_{n}^{T E}$ or $c_{n}^{T M}$, which depend on the size and material properties of the scattering sphere. Due to the symmetry, these coefficients do not depend on the azimuthal order $m$ for spherical scatterers and they may be written in exact closed forms by applying the suitable boundary conditions of the problem at hand [14-15]. For a homogenous spherical scatterer of radius $a$, permittivity $\varepsilon$ and permeability $\mu_{0}$ (assumed the same as that of the background medium), their expressions become:

$$
\begin{gathered}
c_{n}^{T M}=-\frac{\varepsilon j_{n}(k a)\left[\left(k_{0} a\right) j_{n}\left(k_{0} a\right)\right]^{\prime}-\varepsilon_{0}\left[(k a) j_{n}(k a)\right]^{\prime} j_{n}\left(k_{0} a\right)}{\varepsilon j_{n}(k a)\left[\left(k_{0} a\right) h_{n}\left(k_{0} a\right)\right]^{\prime}-\varepsilon_{0}\left[(k a) j_{n}(k a)\right]^{\prime} h_{n}\left(k_{0} a\right)}, \\
c_{n}^{T E}=-\frac{j_{n}(k a)\left[\left(k_{0} a\right) j_{n}\left(k_{0} a\right)\right]^{\prime}-\left[(k a) j_{n}(k a)\right]^{\prime} j_{n}\left(k_{0} a\right)}{j_{n}(k a)\left[\left(k_{0} a\right) h_{n}\left(k_{0} a\right)\right]^{\prime}-\left[(k a) j_{n}(k a)\right]^{\prime} h_{n}\left(k_{0} a\right)}
\end{gathered}
$$

where $k=\omega \sqrt{\varepsilon \mu_{0}}$ and $k_{0}=\omega \sqrt{\varepsilon_{0} \mu_{0}}$ are, respectively, the wave-numbers inside and outside the sphere, and the derivatives are taken with respect to the argument. For passive particles and propagating waves impinging on the object, power conservation requires that $\left|c_{n m}\right| \leq 1$, with the equality obtained at the resonance of a given multipolar order in the ideal lossless case (i.e., when no absorption is present), which happens for the two polarizations when:

$$
\begin{array}{ll}
T M: & \varepsilon j_{n}(k a)\left[\left(k_{0} a\right) y_{n}\left(k_{0} a\right)\right]^{\prime}=\varepsilon_{0}\left[(k a) j_{n}(k a)\right]^{\prime} y_{n}\left(k_{0} a\right), \\
T E: & j_{n}(k a)\left[\left(k_{0} a\right) y_{n}\left(k_{0} a\right)\right]^{\prime}=\left[(k a) j_{n}(k a)\right]^{\prime} y_{n}\left(k_{0} a\right)
\end{array},
$$

with $y_{n}$ being the spherical Neumann function. 
As also discussed in [15], the use of plasmonic materials or metamaterials allows satisfying the conditions for resonance of a given multipolar order $n$ in a sub-wavelength scale, in principle independently of how small the particle is, as long as losses are not considered. For the case at hand Eq. (4) is simplified in the small-radii limit to the well known approximate dispersion condition [1]:

$$
\varepsilon=-\frac{n+1}{n} \varepsilon_{0},
$$

valid only for $T M^{r}$ resonances. As reported in [16], such approximation and the possibility of exciting higher-order resonances in sub-wavelength systems hold as long as the absorption in the particle is negligible, which quantitatively may be written as:

$$
\operatorname{Im}\left[\varepsilon / \varepsilon_{0}\right] \ll\left(k_{0} a\right)^{2 n+1} \frac{n+1}{n^{2}[(2 n-1) ! !]^{2}} .
$$

This condition on the material absorption shows that exciting higher-order resonances in a small sphere is challenging, unless extremely low-loss plasmonic materials are available or their size is adequately increased.

The first-order resonance (for $n=1$ ), which is the one studied and analyzed for many decades, is obtained approximately when $\varepsilon \simeq-2 \varepsilon_{0}$, following (5). This resonance is indeed the main mechanism behind many anomalous physical phenomena in plasmonic optics [1-2], since, among all the resonant orders, it is by far relatively less sensitive to material losses, as Eq. (6) ensures. Even though for this low-order resonance the scattering pattern has the same shape as the one of a classic non-plasmonic dielectric sphere, dominated by its dipolar angular distribution, the total scattered power by a small 
nano-sphere satisfying the resonant condition (5) is comparable with that of a much larger sphere, since the $T M^{r}{ }_{1 m}$ scattered harmonics are excited at their maximum possible amplitude. When moderate losses are added, this resonance also explains the increase of absorbed power by systems of plasmonic particles at their resonant frequencies.

The classic question of how a metallic nanoparticle at its first plasmonic resonance may scatter (or absorb in the case where material losses are present) more power than what is "incident" [17] on it has been addressed in a seminal work of Bohren [11] more than twenty years ago. In his paper, the approximate near-field power flow lines were evaluated by considering only the electric dipolar Mie harmonic, and subsequently a first order Taylor expansion for the spherical Bessel functions (small-radius approximation) around the origin were presented assuming $\varepsilon=-2 \varepsilon_{0}$. The analysis was limited to the region outside the metallic nanoparticle, since the work in [11] was mainly aimed at visually showing the effective increase in the particle scattering cross section, and indeed it was shown, how the effective "cross section" of "rays" that are bent by the presence of the resonant nanoparticle may become sensibly larger than the geometrical or physical cross section of the particle itself, and how some of the rays may hit the particle even on the back of the particle, where shadow is expected. This may provide a physically heuristic explanation of the counterintuitive phenomenon of plasmonic resonance of metallic nanoparticles. Subsequent works have studied this problem in more details, considering more scattering coefficients involved in the near-field problem, in order to better explain the field behavior around the nanoparticle [12-13]. In particular, in [12] it is claimed that higher-order multipoles are needed to evaluate the correct near-field 
power flow at the first (dipolar) resonance, partially correcting the results of [11], whereas in [13] more complex particle shapes were analyzed with full-wave numerical simulations, evidencing interesting vortices and "whirlpools" of power around the resonant particles.

Studying the results presented in [12], it was not clear to us why, at the resonance of the first-order Mie coefficient, the other spherical harmonics (different from the dipolar one) may provide such a drastic contribution to the power flow, despite the well-known fact that at the dipolar resonance, the dipolar terms should be the dominant contributions to the near and far fields. Motivated by this question, in this manuscript we revisit this problem by applying the full-wave Mie scattering theory to analyze the exact (i.e., non approximate) near-field power flow inside and around such nanoparticles at and near their plasmonic resonances, particularly for the higher-order resonances. We demonstrate that employing the exact analytical expressions for the fields of each spherical harmonic of the resonant order by itself, and not their approximate Taylor expansion, is sufficient to describe accurately the power flow around the nanoparticle at its various plasmonic resonances. The results that we report in the following provide physically relevant understanding and clear intuitive insights into enhanced scattering and absorption by plasmonic sub-wavelength particles at their multipolar resonances. We then concentrate on higher-order resonances, studying the power flow inside and around nano-spheres resonant for larger orders of $n$ (i.e., $2^{\text {nd }}$ and $3^{\text {rd }}$ orders). As discussed in detail in [9], the excitation of such resonances may provide the possibility of having super-directive nanoantennas at infrared and optical frequencies, utilizing noble metals or other plasmonic materials. Here we explore how such higher order resonances may be established in a 
deep subwavelength particle and how their power flow behave in the very near-field and inside the particles at these higher-order resonances.

\section{Dipolar resonance: Revisited}

As done in [12-14], the local power flow inside and around the particles may be evaluated by considering the direction of the local Poynting vector $\mathbf{S}=\frac{1}{2} \operatorname{Re}\left[\mathbf{E} \times \mathbf{H}^{*}\right]$, representing the averaged local power flux density. In evaluating $\mathbf{S}$ the local electric $\mathbf{E}$ and magnetic $\mathbf{H}$ fields are calculated using the series expansions (1)-(2) for the external fields, and similar expansions for the fields inside the particle. For the purpose of numerical evaluation, the series are truncated after a certain order $N_{\max }$, which depends on the particle's size and how close to the full resonance of the particle we are, as we discuss in the following.

As the first example, Fig. 1 shows the power flow pattern for a particle with $k_{0} a=0.3$. The particle permittivity is chosen to be tuned at the exact full dipolar resonance predicted by (4), i.e., at the frequency $f$ for which $\varepsilon=-2.223 \varepsilon_{0}$, which happens for silver at $f=837 \mathrm{THz} \quad\left(\lambda_{0}=358 \mathrm{~nm}\right) \quad[18]$ and for aluminum at $f=2021 \mathrm{THz}\left(\lambda_{0}=141 \mathrm{~nm}\right)$ [19]. Realistically, the examples of this section may therefore correspond to the case of a silver particle of $17 \mathrm{~nm}$ radius. Moreover, in the case of Fig. 1, the presence of absorption and losses has been neglected for now (which for both $A g$ and $A l$ would be about $\operatorname{Im}[\varepsilon]=0.2)$. This is considered in the next example. We point out that the size of the particle has been deliberately chosen to be the same as the one considered in [11-12]. The excitation consists of a plane wave traveling 
along the positive $z$ axis with electric field linearly polarized along $x$. In this way, for the reason of symmetry, only the spherical harmonic with $m=1$ are excited and all multipolar orders $n$ may be in principle excited by this form of excitation. For the particular choice of material permittivity, we get the scattering coefficient $c_{1}^{T M}=-1$, consistent with the full dipolar resonance of the particle [15], and the relative amplitude of the other scattering orders is: $\left|c_{2}^{T M}\right|=3.8 \cdot 10^{-4}\left|c_{1}^{T M}\right|,\left|c_{1}^{T E}\right|=1.7 \cdot 10^{-4}\left|c_{1}^{T M}\right|$, with the other scattering coefficients being negligible.

It is therefore clear that the near-field distribution, as well as the far-zone field, is entirely dominated by the dipolar resonance. As a result, it is sufficient to consider the interaction between the impinging plane wave and the $T M_{11}$ spherical harmonic in order to evaluate the near-field power flow outside the particle. This is different in some sense from what was claimed in [12], where it was argued that the first-order Mie term may not be adequate to describe accurately such a power flow. In that work, however, the Taylor expansion of the scattering coefficients was considered in order to determine the power flow lines, and in the frame of that approximation one needed to consider higher-order terms, since the approximate dipolar term is not adequate, particularly in the neighborhood of the full resonance of the system, to properly describe the field behavior. The inadequacy of this approximation in the case at hand is also evident in the difference between the exact resonance of such a sphere as predicted by (4) for $\varepsilon=-2.223 \varepsilon_{0}$, and the approximate expression $\varepsilon=-2 \varepsilon_{0}$ obtainable from (5). As we have found in our calculation, we reiterate that in this case and for any other sub-wavelength particle, it is sufficient to truncate the scattered field expansion to just the resonant order, and the 
power flow is therefore substantially given by the interaction of the resonant spherical harmonic with the impinging field. This provides the power flow distribution depicted in Fig. 1, which is in many aspects different from those predicted in [12] for the analogous electromagnetic problem (lossless particle at the dipolar resonance).

For the field inside the particle, due to the limited size of the objects considered here, the first two $T E^{r}$ and $T M^{r}$ spherical harmonics are generally more than sufficient to obtain total convergence of the fields and power flow. The spherical Bessel functions $j_{n}\left(k_{0} r\right)$, in fact, decay near the origin as $\frac{2^{n} n !\left(k_{0} r\right)^{n}}{(2 n+1) !}$ [20], and although the expansion coefficients for the field inside the particle decay more slowly than the corresponding scattering coefficients when the order $n$ is increased, the contributions from spherical harmonics higher than the first few orders are negligible for the inside field.

The power lines of Fig. 1a correspond to the E plane, whereas Fig. 1b depicts the H plane distribution. They are obtained considering the contributions from the combination of the first three orders of $T E^{r}$ and $T M^{r}$ spherical harmonics together with the exact expression for the fields of the incident plane wave. As already anticipated, convergence is achieved after just considering the resonant $T M_{11}$ harmonic for the outside scattered field and the first two harmonics for the inner field. Interesting peculiarities for the power flow inside and outside the particle are highlighted by these plots.

As already predicted in [11], the plane wave cross section that is effectively perturbed by the presence of the resonant particle is much larger than the effective geometrical cross section of the particle itself (the figure shows an area of $16 a \times 16 a$ around the particle). The power flow lines tend to converge towards the particle in the E plane and to circulate 
around it (Fig. 1a), whereas they seem to diverge away from the particle in the $\mathrm{H}$ plane (Fig. 1b). It is interesting to note that in both planes a significant portion of the power is entering from the back of the particle near the $z$ axis, which might otherwise be expected to be in a "shadow" region. The power substantially enters the particle only from a small solid angular region around the $z$ axis in the front and in the back of the particle in the $\mathrm{E}$ plane, and it circulates around the particle in the remaining portion of the sphere, consistent to what was obtained in [12-13]. In the E plane (Fig. 1a) two saddle points in the front and in the back of the particle on the $z$ axis are seen where the power seems to converge before circulating around the particle. Although the Poynting vector is rigorously tangential to the plane both in the $\mathrm{E}$ and in the $\mathrm{H}$ plane, in these singular points the power is collected from, or redirected to, the direction orthogonal to the planes, and thus feeds specific resonant paths. Also a saddle curve is seen in the E plane, running horizontally along the $x$ axis, with the power being collected along this line inside the particle and redirected outside the particle from this line. These saddle lines and saddle points act as "sources" or "sinks" in the planes depicted here, since in such singular loci the power can effectively flow in or out along the direction orthogonal to the plane. In the $\mathrm{H}$ plane, similar saddle points and saddle curves are also noticeable, and two vortices of power lines are present on the two sides of the particle. The power flow is indeed very complicated and manifests the presence of a strong resonant phenomenon in which the power feeds the particle and interacts in tight loops and vortices with specific patterns. We are indeed experiencing a full resonance of an open cavity with circulation of power flow that is relatively higher than what is impinging on the particle. This explains how the total scattering cross section for the case at hand is enhanced with a factor of 
$Q_{s c a}=66.67$ with respect to the geometrical cross section of the nano-sphere. This is indeed consistent with the size of the "effective" area involved in this resonant process, whose averaged radius is about eight times larger than the particle radius on both planes. As already outlined, we notice that this exact configuration of power flow lines is indeed different from the one reported in [12], even for the same lossless resonant particle that is considered here. This is due to the fact that at the full resonance of the structure the "quasi-static" Taylor approximations of spherical harmonics and scattering coefficients considered in [12] are not adequate to fully predict the field behavior, particularly in the near-field of the structure.

The power flow inside the sphere also presents interesting peculiarities. The pattern is caused by the interaction of the low-order spherical harmonics excited inside the particle (we note that each spherical harmonic by itself does not carry any real power and $\mathbf{S}$ is identically zero, since each harmonic is represented by a standing wave $\left.j_{n}(k r)\right)$. The power flow converges towards the saddle curve cutting the particle along the $x$ axis in the E plane, flowing in the orthogonal direction, and along the $y$ axis outside the sphere in the $\mathrm{H}$ plane. The power flow from inside the particle feeds the outer resonant distribution and it exits the sphere from the equatorial $x-y$ plane. Also this anomalous power flow inside the particle, showing a complex vortex flow linking the two planes, is distinct from the results available in the literature.

Fig. 2 considers the same geometry as in Fig. 1, but adding now the material loss in the amount already considered in [11-12], i.e., $\varepsilon=(-2.223+i 0.2) \varepsilon_{0}$, which is consistent with the absorption level of $A g$ or $A l$ [18-19]. In this case, some of the saddle points and vortices in the two planes disappear, due to the absorption inside the particle. The 
pattern in the E plane is similar to the one predicted in [11] considering just the first order Taylor expansion of the dipolar harmonic (surprisingly different in both the $\mathrm{E}$ and $\mathrm{H}$ planes from the one obtained in [12] for this same level of losses using additional Taylor polynomials [21]). An anomalous circulation of power is still evident and a saddle point on the back of the particle is present in both planes, which effectively connects the two planes. A significant amount of power is again entering from the back of the particle, consistent with the finding of [11]. The angular window around the $z$ axis from where the power enters the particle gets larger when losses are considered. For this case the scattering efficiency is lowered to $Q_{s c a}=3.7$, whereas the absorption efficiency is $Q_{a b s}=12$. Inside the nanoparticle, the presence of a saddle line where the power flows from the $\mathrm{E}$ to the $\mathrm{H}$ plane is still evident (similar to the lossless case). These findings are distinct from what reported in [13], and show further complexity and interesting behavior in the nano-vortices near and inside resonant plasmonic nano-particles.

\section{Higher-order resonances}

Having revisited the anomalous power flow behavior for the dipolar resonance of a subwavelength plasmonic nanoparticle, it is of interest now to analyze the behavior of higher-order resonances in terms of their power flow distribution. As noticed in [9], the higher-order plasmonic resonances are characterized by a high variation of the fields inside and around the particle. As envisioned in [9], the possibility of exciting and establishing a spatially rapidly varying electromagnetic field distribution in a nano-scale domain may in principle allow the realization of highly directive nano-antennas with a sharp beam, without a need for electrically large antenna arrays and/or complicated feed networks. Sensitivity of such higher-order resonances to material losses increases 
dramatically with the order $n$, as Eq. (6) shows. Some experimental evidence of quadrupolar excitations in metallic nano-particles has been reported in the literature [22]. We also note that Eq. (6) depends on the size of the particle. Therefore, higher tolerance to material loss in a higher-order resonance may be achieved by increasing the particle size.

In Fig. 3, the same ideally lossless metallic nano-sphere of Fig. 1 is assumed to be excited at a frequency for which $\varepsilon=-1.533 \varepsilon_{0}$, which corresponds to the full resonance of the $T M_{21}$ (electric quadrupolar) spherical harmonic, as predicted by (4). For this case, the scattering coefficient $c_{2}^{T M}=-1$ and $\left|c_{1}^{T M}\right|=6.96 \cdot 10^{-2}\left|c_{2}^{T M}\right|,\left|c_{1}^{T E}\right|=1.3 \cdot 10^{-4}\left|c_{2}^{T M}\right|$, and the other scattering coefficients are negligible. Again, the convergence of the near-field and power flow lines is obtained by just considering the full-wave second order $T M_{21}$ spherical harmonic. In both E plane (Fig. 3a) and H plane (Fig. 3b) an intricate pattern of vortices, saddle lines and complicated power flow distribution is established inside and around the particle. The region perturbed by the resonance is larger than the one obtained in Fig. 1 for the dipolar resonance, consistent with the fact that the scattering efficiency is now increased to $Q_{s c a}=111.4$, following the formula $Q_{s c a}=\frac{2(2 n+1)}{\left(k_{0} a\right)^{2}}$, derived in [15] for any order of resonance. In the E plane, one saddle point is located far on the back of the particle and another one still on the back, but close to its surface, both along the $z$ axis. Another two saddle points arise in the same plane on the $x$ axis, again very close to the plasmonic particle's interface. The power flow lines show an interesting subwavelength vortex pattern around the particle that connects the different saddle points. Inside the particle, the distribution is also very intricate, showing that the power enters 
the nano-sphere only from a very narrow angular region around the $z$ axis and exits from another narrow region around the $x$ axis. A multiple number of saddle lines are observed inside the particle, making even more complex the power flow vortices and the power exchange between orthogonal planes.

The $\mathrm{H}$ plane also shows a complex pattern of saddle lines, one running along the $y$ axis inside and outside the particle and closing into a saddle point in front of the particle on the $z$ axis. The $16 a \times 16 a$ area depicted in the two figures does not include all the area perturbed by the resonance, which affects even a larger region, particularly on the back of the particle. This distribution is far from being close to that of the impinging plane wave even at a distance of $8 a$ from the particle center, as seen from Fig. 3.

The complexity of the power flow is related to the rapidly varying near-field distribution associated with these higher-order resonances. For instance, Fig. 4 reports the magnitude of the near-field perpendicular magnetic field to the $\mathrm{E}$ plane and magnetic field on the $\mathrm{H}$ plane for both the quadrupolar and octopolar resonances in such nano-particles. It is evident how the power-flow vortices described in Fig. 3 and the following figures are indeed related to the rapidly varying fields of Fig. 4, also consistent with the findings reported in [9].

Consistent with Fig. 4, the power flow distribution is further complicated when we move to the third-order resonance, which for the same ideal lossless plasmonic particle arises for $\varepsilon=-1.346 \varepsilon_{0}$. The power flow lines for this resonance are depicted in Fig. 5a for the E plane and Fig. $5 \mathrm{~b}$ for the $\mathrm{H}$ plane. In this case $c_{3}^{T M}=-1$ and $\left|c_{1}^{T M}\right|=5 \cdot 10^{-2}\left|c_{3}^{T M}\right|$, $\left|c_{2}^{T M}\right|=10^{-3}\left|c_{3}^{T M}\right|, \quad\left|c_{1}^{T E}\right|=1.2 \cdot 10^{-4}\left|c_{3}^{T M}\right| . \quad$ The scattering efficiency increases to 
$Q_{s c a}=155.73$ and this is reflected in the further increase of the area perturbed by the particle. Multiple vortices are noticeable around the particle in both planes. The complicated power flow resembles and corresponds to the quickly varying near-field configuration for these higher-order resonances, which cause narrow and directive multiple beams in the far-field [9]. This is consistent with the near-field distributions for this case of Fig. 4c and Fig. 4d. Very pronounced saddle lines run all over the near-field in both planes.

Figure 6 shows a zoom of these power flow patterns in a region of space closer to the nanoparticle (the depicted square is now $6 a \times 6 a$ ). The strong vortices generated by the anomalous higher-order resonance are made more evident both inside and outside the particle. There are saddle points that act as sinks and sources in the two planes, where power enters or exits from the plane in the orthogonal direction. In the E plane (Fig. 6a) the power enters the sphere from both the upper and the lower hemisphere around the $z$ axis, feeding a flow of power that runs around the sphere oppositely to the flow running inside the particle. This is consistent with a tight surface plasmon polariton guided around the spherical surface, feeding this intricate vortices, for which the power flows are oppositely oriented on the two sides of the plasmonic interface. A saddle line where power enters and leaves the $\mathrm{E}$ plane runs along the $x$ axis crossing the nano-sphere. Along this line the power enters the plane in the region outside the particle and flows into the orthogonal plane inside the particle. In the $\mathrm{H}$ plane (Fig. 6b) the saddle lines are oblique, with power flowing inside the particle around the $z$ axis and flowing outside the particle towards the two saddle points along the $y$ axis. 
Figure 7 and Fig. 8 report the same results as in Fig. 3 and Fig. 5, respectively, but considering some small losses in the plasmonic material. In particular, Fig. 7 refers to the quadrupolar resonance and the particle is assumed to have a permittivity $\mathcal{E}=\left(-1.533+i 5 \cdot 10^{-4}\right) \varepsilon_{0}$, whereas Fig. 8 refers to the octoplar resonance and the particle has $\varepsilon=\left(-1.346+i 10^{-6}\right) \varepsilon_{0}$. We note that these material losses are much less than the usual realistic losses for a typical noble metal (e.g., silver). However, here we use these small losses, just to conceptually demonstrate how they affect the patterns of power flow. We note how the area perturbed by the resonance has decreased when losses are considered, even though the interesting power vortices are still present around and inside the nano-particle. Further increasing the level of losses towards realistic values would cancel the resonant effects for such sub-wavelength dimensions of this geometry, consistent with Eq. (6).

It is however possible to find a trade-off between size and amount of material losses for envisioning the excitation of these near-field power vortices with realistic optical materials. Another viable solution may be to utilize layered particle (i.e., core-shell nanoparticle), that may allow the simultaneous excitation of a higher-order resonance and the complete suppression of the dipolar pattern, using the technique proposed in [6]. This possibility has been already proposed in [9] for realizing a quadrupolar sub-wavelength nano-antenna relatively more robust to the presence of material losses.

Intuitively speaking, the multi-center nature of these optical power flow patterns is analogous to that of higher order modal structures in cavity resonators. The tight and strong power vortices highlighted in Fig. 6, and consistent with those envisioned for lower-order resonances in section 2 and in [13], may provide an interesting mechanism 
for potential applications at optical or infrared frequencies. One may speculate that these may be employed to move or trap a small nano-particle placed in the vicinity of the resonant sphere. The high level of the fields and of the power flow caused by the strong resonance indeed suggests that a significant electromagnetic stress tensor may be locally applied in the vicinity of the particle. We speculate that the local force caused by these vortices may potentially be useful for some applications, e.g., moving a nano-particle, acting locally as optical tweezers, or for trapping it in a specific location. We note that the presence of small non-resonant nano-objects in the close vicinity of the resonant nanosphere is not expected to perturb significantly the field configurations predicted here, due to the fact that such distribution is strongly resonant and it is dominant over the impinging field and any other non-resonant field pattern superimposed on it. We are currently exploring basic issues for such potential applications.

\section{Acknowledgements}

This work is supported in part by the U.S. Air Force Office of Scientific Research (AFOSR) grant number FA9550-05-1-0442.

\section{References}

[1] C. F. Bohren, and D. R. Huffman, Absorption and Scattering of Light by Small Particles (Wiley, New York, 1983).

[2] M. Kerker, "Founding fathers of light scattering and surface-enhanced Raman scattering," Applied Optics 30, 4699-4705 (1991).

[3] J. B. Pendry, "Negative refraction makes a perfect lens," Physical Review Letters 85, 3966-3969 (2000). 
[4] M. Quinten, A. Leitner, J. R. Krenn, F. R. Aussenegg, "Electromagnetic energy transport via linear chains of silver nanoparticles", Optics Letters 23, 13311333 (1998).

[5] N. Engheta, A. Salandrino, and A. Alù, "Circuit elements at optical frequencies: nano-inductors, nano-capacitors and nano-resistors," Physical Review Letters 95, 095504 (2005).

[6] A. Alù, and N. Engheta, "Achieving transparency with plasmonic and metamaterial coatings," Physical Review E 72, 016623 (2005) [erratum in Physical Review E 73, 019906 (2006)].

[7] M. G. Silveirinha, A. Alù, and N. Engheta, "Parallel plate metamaterials for cloaking structures," Physical Review E 75, 036603 (2007).

[8] A. Alù, and N. Engheta, "Plasmonic materials in transparency and cloaking problems: mechanism, robustness, and physical insights," Optics Express 15, 3318-3332 (2007).

[9] A. Alù, and N. Engheta, "Enhanced directivity from sub-wavelength infrared/optical nano-antennas loaded with plasmonic materials or metamaterials," IEEE Transactions on Antennas and Propagation (to be published).

[10] G. Mie, "Considerations on the optics of turbid media, especially colloidal metal sols," Annalen der Physik 25, 377-442 (1908).

[11] C. F. Bohren, "How can a particle absorb more than the light incident on it?," American Journal of Physics 51, 323-327 (1983). 
[12] Z. B. Wang, B. S. Lukyanchuk, M. H. Hong, Y. Lin, and T. C. Chong, "Energy flow around a small particle investigated by classical Mie theory," Physical Review B 70, 035418 (2004).

[13] M. V. Bashevoy, V. A. Federov, and N. I. Zheludev, "Optical whirlpool on an absorbing metallic nanoparticle,” Optics Express 13, 8372-8379 (2005).

[14] J. A. Stratton, Electromagnetic Theory (McGraw-Hill Comp., New York and London, 1941).

[15] A. Alù, and N. Engheta, "Polarizabilities and effective parameters for collections of spherical nano-particles formed by pairs of concentric doublenegative (DNG), single-negative (SNG) and/or double-positive (DPS) metamaterial layers," Journal of Applied Physics 97, 094310 (2005) [erratum in Journal of Applied Physics 99, 069901 (2006)].

[16] M. I. Tribelskii, "Resonant scattering of light by small particles," Sov. Phys. JETP 59, 534-536 (1984).

[17] By "incident on the particle" the problem is considered in terms of geometrical optics.

[18] P. B. Johnson, and R. W. Christy, "Optical constants of the noble metals,"Phys. Rev. B 6, 4370-4379 (1972).

[19] D. Bohm, and E. P. Gross, "Theory of plasma oscillations. A. Origin of medium-like behavior ," Phys. Rev. 75, 1851-1864 (1949).

[20] M. Abramowitz, and I. A. Stegun, (Eds.). "Spherical Bessel Functions." §10.1 in Handbook of Mathematical Functions with Formulas, Graphs, and Mathematical Tables, (9th printing. New York: Dover, pp. 437-442, 1972). 
[21] We note that the analysis in [12] expands the whole scattered field in the Taylor approximation, mixing together the different Mie spherical harmonics. Although this procedure eventually ensures numerical convergence, it requires a much higher number of terms in order to predict the correct results near the resonance of the nano-particle, similar to the cases considered here. This explains the disagreement between our full-wave results and the approximate calculation in [12]. Also contrary to the claim mentioned in [12] that a correct evaluation of the power flow distribution would require multiple Mie orders, we believe that this is not the case, in the sense that, even if in their Taylor expansion higher-order terms are needed, with a full-wave Mie harmonic expansion one would require one single resonant harmonic to predict the overall power distribution, as it has been shown here.

[22] S. J. Oldenburg, G. D. Hale, J. B. Jackson, and N. J. Halas, "Light scattering from dipole and quadrupole nanoshell antennas," Applied Physics Letters 75, 1063-1065 (1999). 


\section{List of Captions}

Figure 1 - (Color online) Power flow around a spherical nano-particle with $\varepsilon=-2.223 \varepsilon_{0}$, $k_{0} a=0.3$, under plane wave incidence at its first resonance in the E plane (a) and $\mathrm{H}$ plane (b). Brighter (darker in the grayscale version) arrows correspond to higher values of power density flow.

Figure 2 - (Color online) The same as in Fig.1, but with material loss included. Here, the spherical metallic nano-particle has permittivity $\varepsilon=(-2.223+i 0.2) \varepsilon_{0}, \quad k_{0} a=0.3$. Brighter (darker in the grayscale version) arrows correspond to higher values of power density flow.

Figure 3 - (Color online) Power flow around a spherical nano-particle with $\varepsilon=-1.533 \varepsilon_{0}$, $k_{0} a=0.3$, under plane wave incidence at its second (i.e., quadrupolar) resonance in the $\mathrm{E}$ plane (a) and $\mathrm{H}$ plane (b). Brighter (darker in the grayscale version) arrows correspond to higher values of power density flow.

Figure 4 - (Color online) Amplitude of the perpendicular magnetic field distribution in the E plane (a) and of the electric field distribution on the $\mathrm{H}$ plane (b) for the nanoparticle at its quadrupolar resonance, corresponding to the geometry of Fig. 3. Panels c) and d) correspond to the octopolar resonance, for the geometry of Fig. 5-6. Brighter (darker in the grayscale version) colors correspond to higher values of the field amplitude. 
Figure 5 - (Color online) Power flow around a spherical nano-particle with $\varepsilon=-1.346 \varepsilon_{0}, k_{0} a=0.3$, under plane wave incidence at its third (octopolar) resonance in the E plane (a) and $\mathrm{H}$ plane (b). Brighter (darker in the grayscale version) arrows correspond to higher values of power density flow.

Figure $6-$ (Color online) The same as in Fig. 5, but for a region of space closer to the nano-sphere (here the total computational plane is $6 \lambda_{0} \times 6 \lambda_{0}$ ). Again, a) depicts the $\mathrm{E}$ plane and b) the $\mathrm{H}$ plane. Brighter (darker in the grayscale version) arrows correspond to higher values of power density flow.

Figure 7 - (Color online) The same as in Fig. 3, but with small material loss included. Here, the spherical metallic nano-particle has permittivity $\varepsilon=\left(-1.533+i 5 \cdot 10^{-4}\right) \varepsilon_{0}$, $k_{0} a=0.3$. Brighter (darker in the grayscale version) arrows correspond to higher values of power density flow.

Figure 8 - (Color online) The same as in Fig. 5, but with material loss included. Here, the spherical metallic nano-particle has permittivity $\varepsilon=\left(-1.346+i 10^{-6}\right) \varepsilon_{0}, \quad k_{0} a=0.3$. Brighter (darker in the grayscale version) arrows correspond to higher values of power density flow. 
Figures

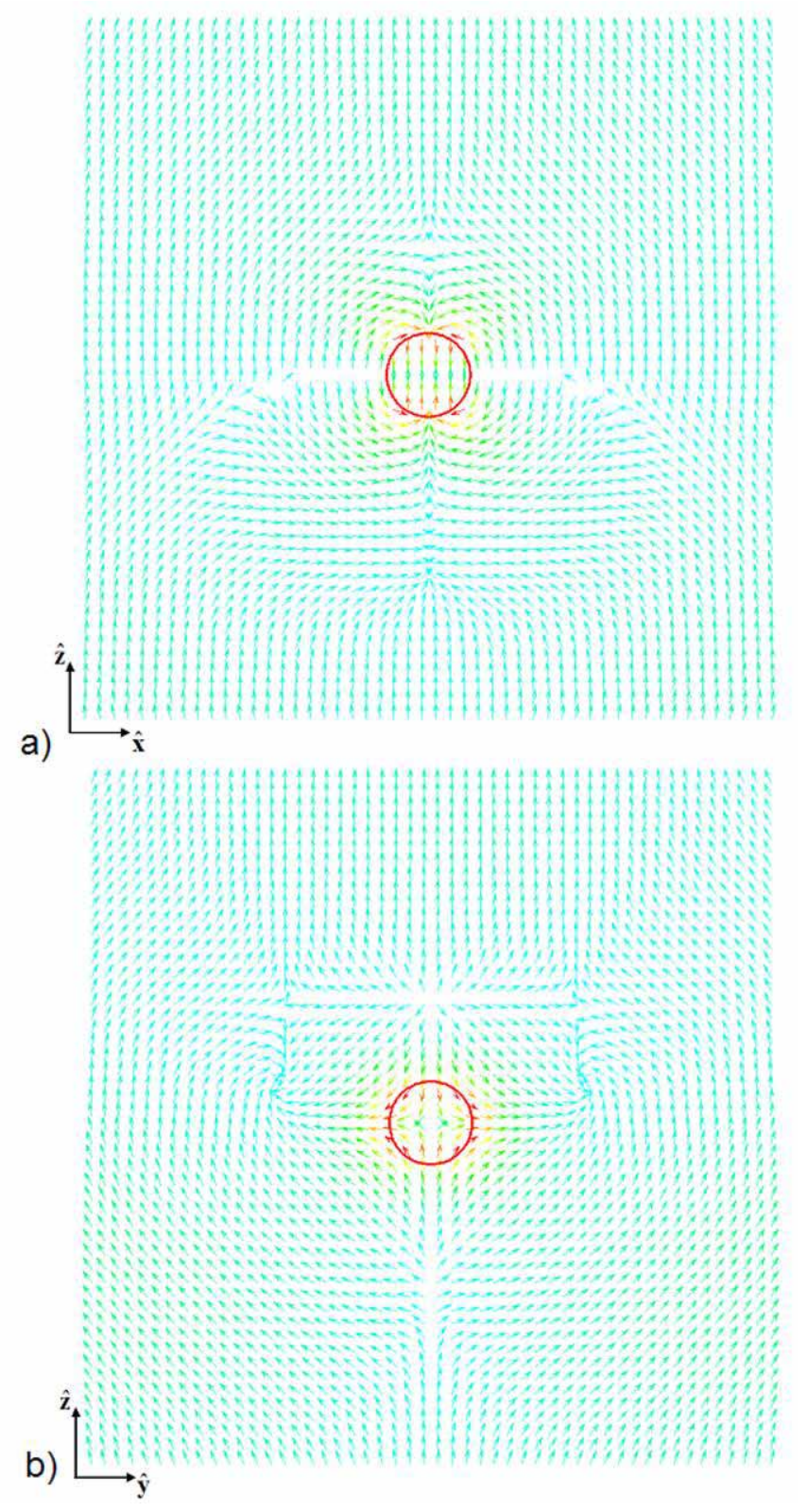

Figure 1 


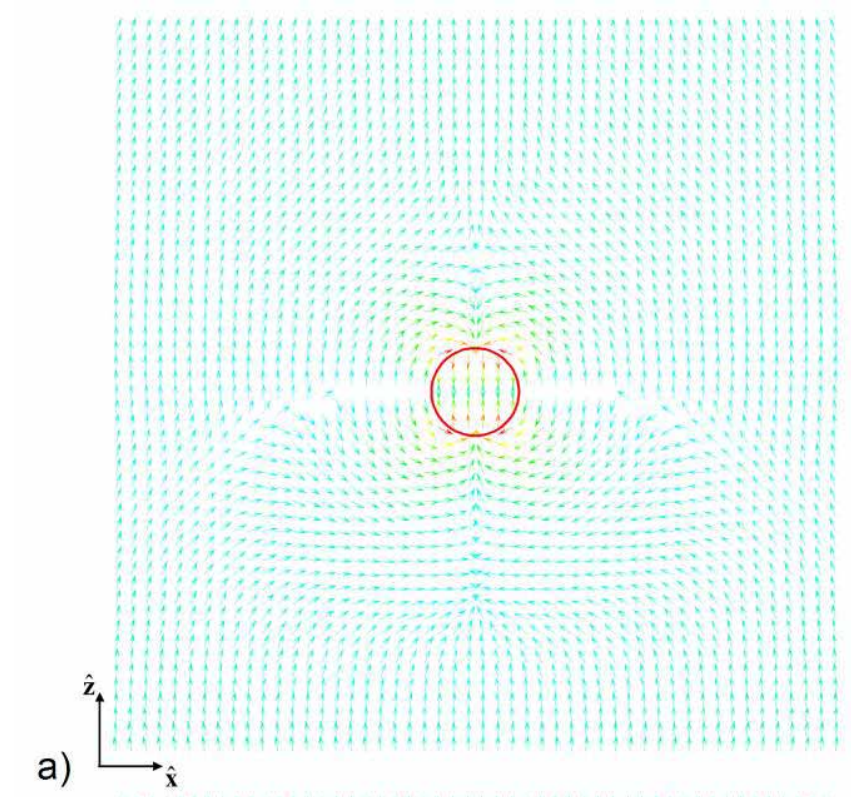

a)

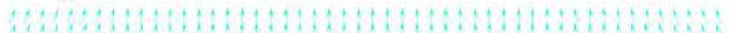

b) $\longrightarrow \hat{y}$

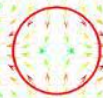

Figure 2 


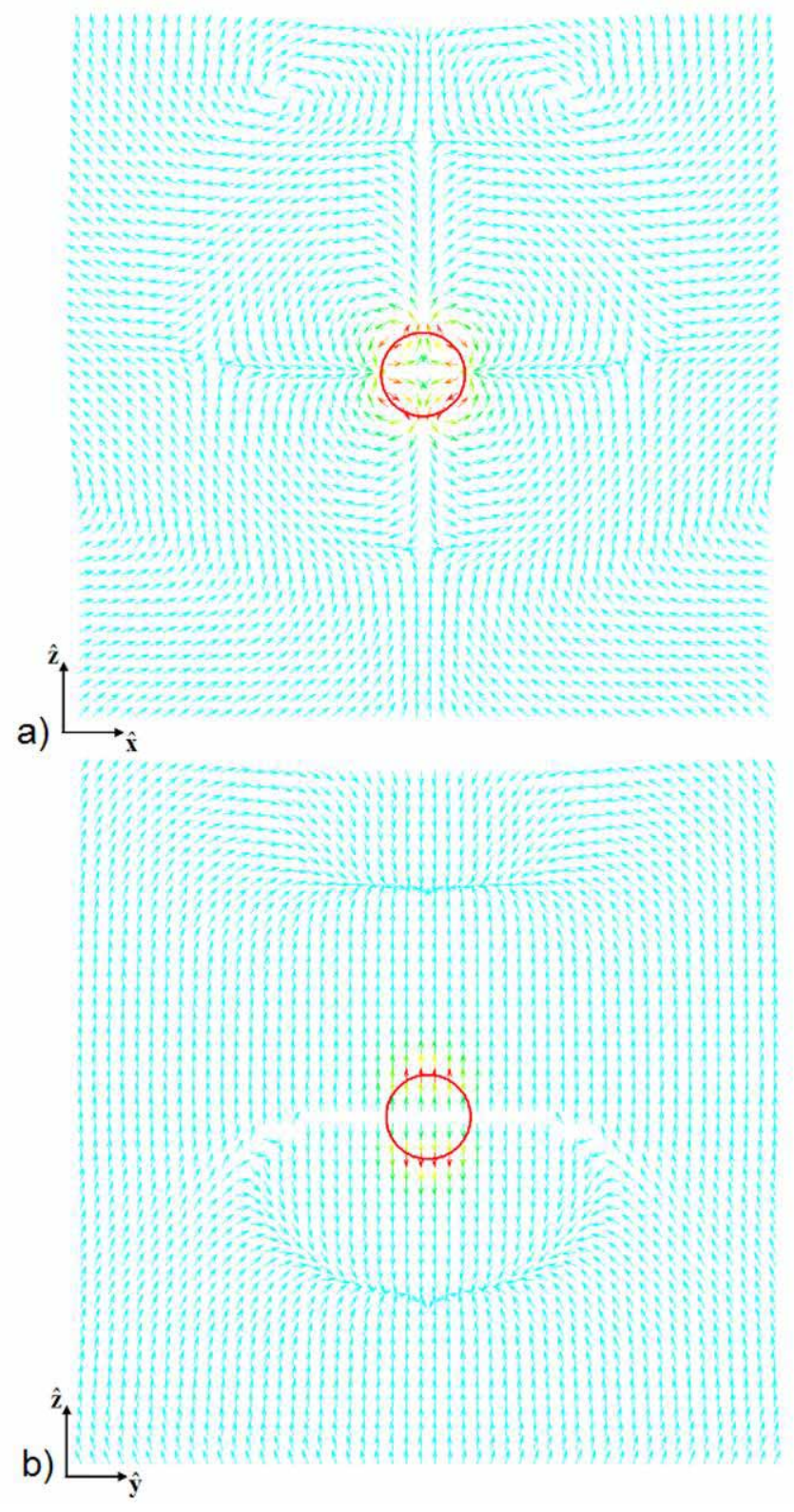

Figure 3 

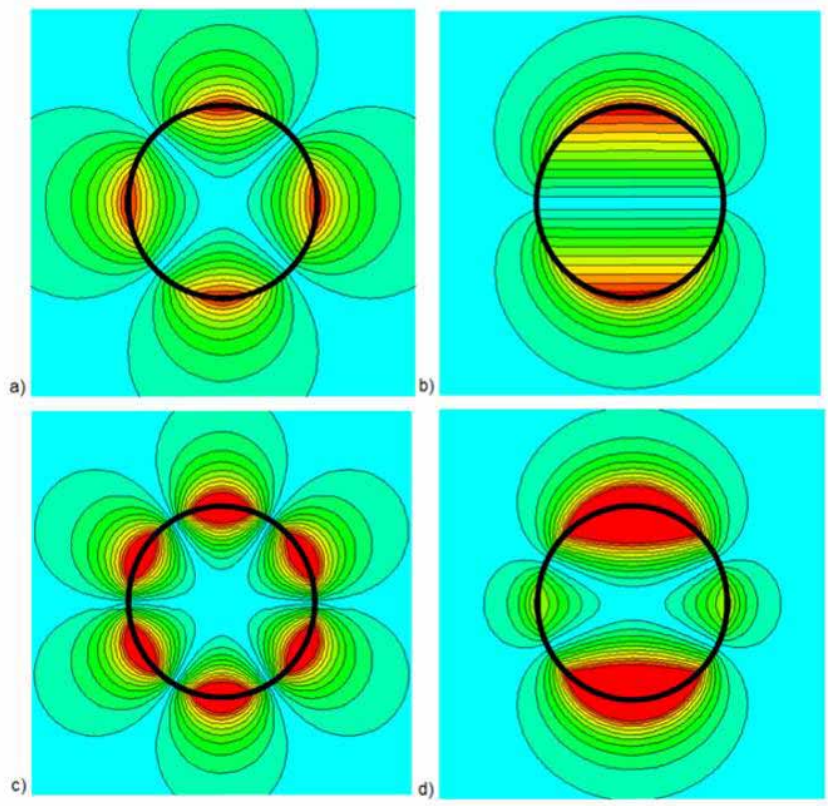

Figure 4 


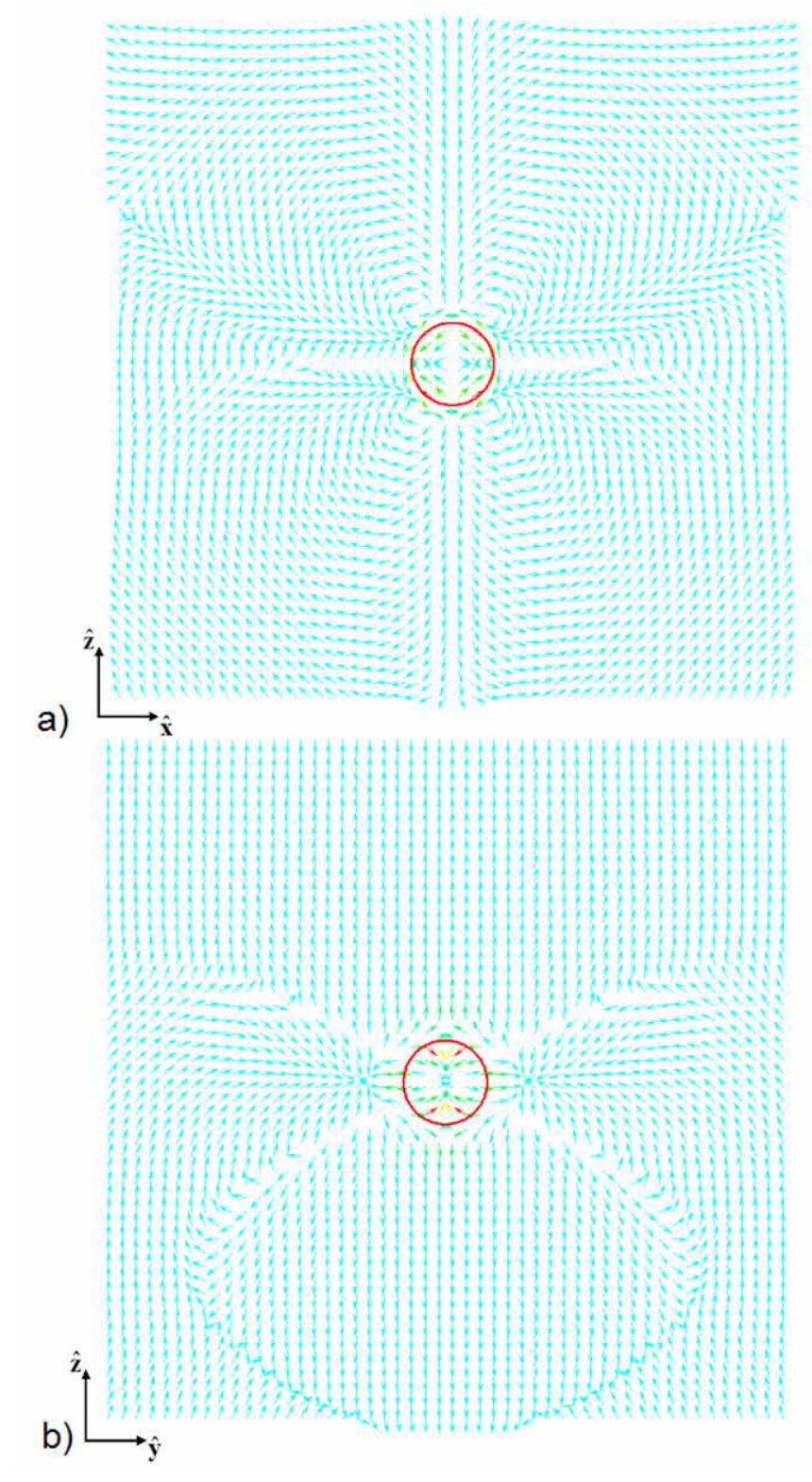

Figure 5 


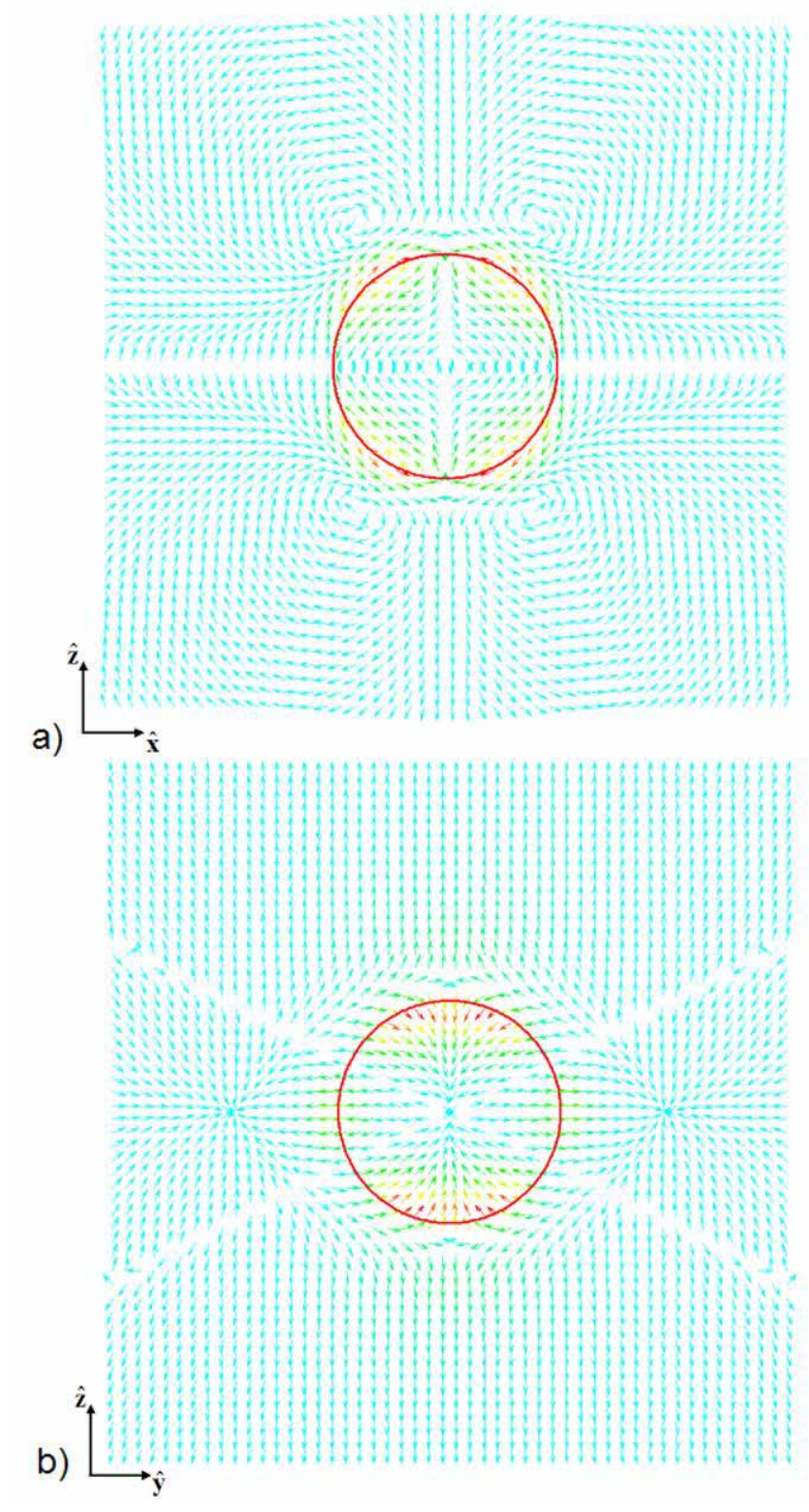

Figure 6 


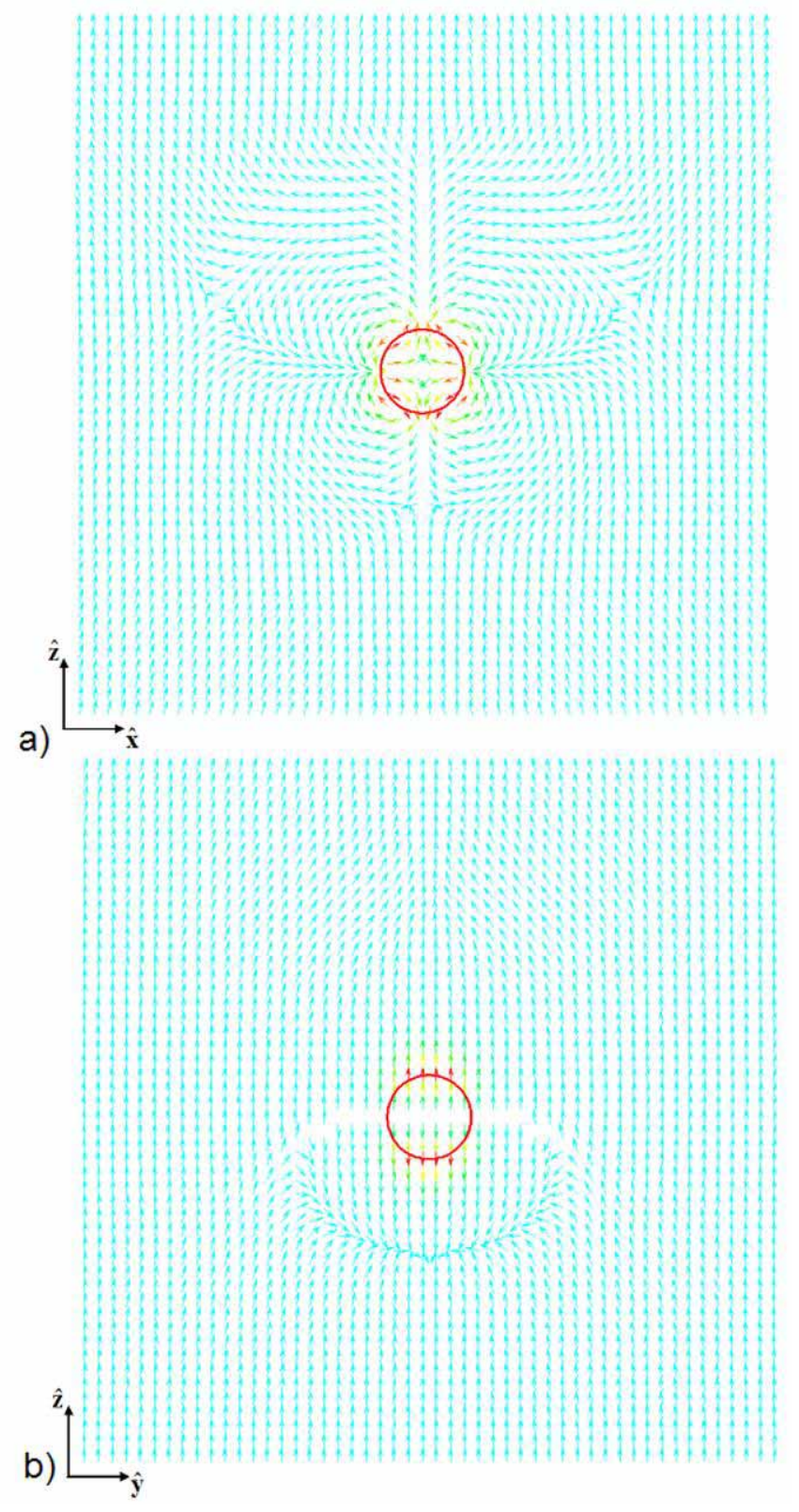

Figure 7 


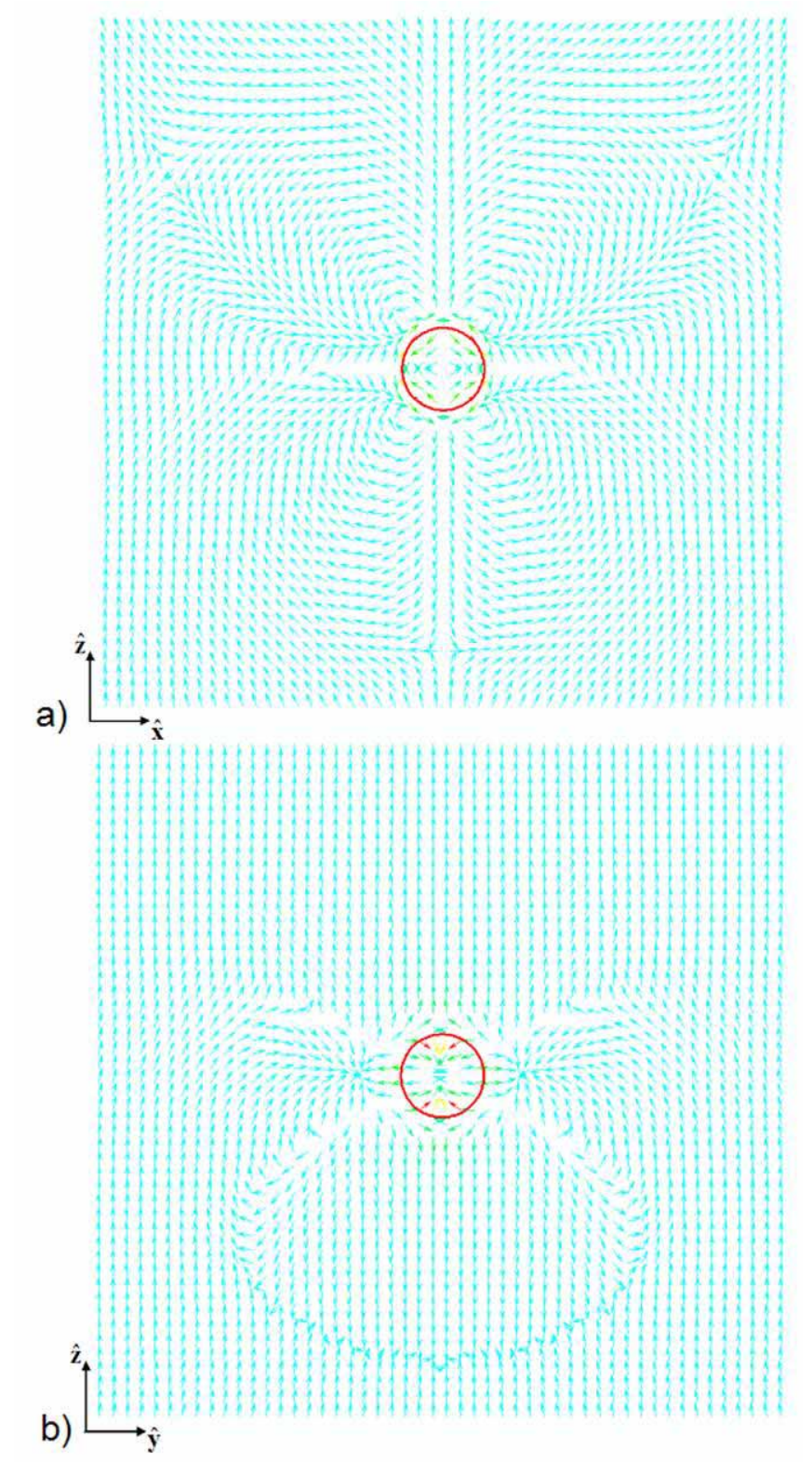

Figure 8 